\title{
ROUTINE NEONATAL EAR CANAL CULTURES ARE NOT USEFUL PREDICTORS OF
} BLOODSTREAM INFECTION (BSI)

\author{
A. Battersby ${ }^{1,2}$, R. Webster ${ }^{2,3}$, T. Neal ${ }^{3}$, N. Subhedar ${ }^{2}$ \\ ${ }^{1}$ Neonatal Medicine, Imperial College London, London, ${ }^{2}$ Neonatal Unit, Liverpool Women's Hospital, \\ ${ }^{3}$ Department of Medical Microbiology, Royal Liverpool University Hospital, Liverpool, UK
}

Background and aims: Culture of body surfaces including the ear canal, throat and rectum are often routinely performed on new neonatal unit admissions. The evidence base for this practice is limited. The aims of the study were:

(1) To identify whether organisms isolated from the ear canal are responsible for blood stream infection (BSI).

(2) To ascertain whether ear canal cultures identify bacteria distinct from those in the throat and rectum.

Methods: We identified infants admitted to the level one neonatal unit over a six month period (AprilSeptember 2008). Clinical and microbial data were obtained from the "Badger" electronic patient database and the "Telepath" microbiology database respectively.

Results: 601 infants were included. 66\% (397/601) had ear canal cultures performed and 30.5\% (121/397) were positive. $6.5 \%(24 / 397)$ of those with ear canal cultures developed blood culture positive early onset BSI. The ear canals of 12.5\% (3/24) of infants harboured the same organisms as isolated from the blood: 2 coagulase negative staphylococci and 1 group B streptococcus which were also isolated from the throat and rectum. 3\% (3/121) of infants had organisms isolated from the ear canal but not the throat or rectum, but none developed culture positive BSI.

Conclusions: On the basis of this small study, the neonatal ear canal does not appear to harbour clinically significant organisms distinct from those in the throat or rectum. Ear canal cultures are not helpful in predicting the organisms responsible for BSI and their acquisition should not form part of routine clinical practice. 